\title{
Dynamical Seasonal Prediction of Climate Extremes in the Pacific
}

\author{
$\underline{\text { A. Charles }}{ }^{\text {a }}$ E. Miles , ${ }^{\mathrm{b}}$ A. Griesser ${ }^{\mathrm{b}}$, R. de Wit ${ }^{\mathrm{a}}$, K. Shelton ${ }^{\mathrm{b}}$, A. Cottrill ${ }^{\mathrm{b}}$, C. Spillman ${ }^{\mathrm{b}}$, \\ H. Hendon ${ }^{\mathrm{b}}$, P. McIntosh , T. Nakaegawa , ${ }^{\mathrm{c}}$ T. Atalifo, ${ }^{\mathrm{d}}$ B. Prakash ${ }^{\mathrm{d}}$, S. Seuseu , ${ }^{\mathrm{e}}$ S. Nihmei , ${ }^{\mathrm{fg}}$ \\ J.Church , ${ }^{\mathrm{h}}$ D. Jones ${ }^{\mathrm{a}}$ and Y. Kuleshov ${ }^{\mathrm{a}}$ \\ ${ }^{a}$ National Climate Centre, Bureau of Meteorology, Melbourne, Australia \\ ${ }^{\mathrm{b}}$ Centre for Australian Weather and Climate Research, Bureau of Meteorology, Melbourne, Australia \\ ${ }^{\mathrm{c}}$ Meteorological Research Institute, Japan Meteorological Agency, Tsukuba, Japan \\ ${ }^{\mathrm{d}}$ RSMC Nadi, Fiji Meteorological Service, Fiji \\ eSamoa Meteorology Division, Apia, Samoa \\ ${ }^{\mathrm{f}}$ Vanuatu Meteorological and Geo-Hazard Department, Port Vila, Vanuatu \\ ${ }^{g}$ Secretariat of the Pacific Regional Environment Programm (SPREP), Apia, Samoa \\ ${ }^{\mathrm{h}}$ Commonwealth Scientific and Industrial Research Organisation, Hobart, Australia \\ Email: a.charles@bom.gov.au
}

\begin{abstract}
The vulnerability of Pacific Island Countries to climate extremes is exacerbated by a changing climate. Many of the early impacts of climate change are being felt through seasonal variability. Drought and coastal inundation are two examples of events which occur as seasonal variability but are made more severe by increased mean temperatures and rising sea levels. Early warnings of extreme climate events from dynamical coupled ocean-atmosphere global climate models (CGCMs) can increase resilience by providing a lead time in which action can be taken.

Dynamical seasonal forecasting systems are now competitive with the best statistical forecasting techniques, with the advantage that they implicitly include the effects of a changing climate, reducing concerns about the effects of climate change and decadal variability on forecasts. Dynamical models have the advantage of providing information at a range of timescales from weeks to seasons and for a range of physical quantities including extreme ocean temperatures, tropical cyclone activity and sea level.

We have developed dynamical model based seasonal forecasts for Pacific Island Countries, including monthly to seasonal forecasts of extreme sea levels, extreme ocean temperatures and tropical cyclone activity. These new forecasts of extreme climate events work in concert with more traditional seasonal outlooks for rainfall, temperature and climate indices to provide rich foresight of near-future climate variability.

Outlooks are delivered via interactive web-based geospatial apps, providing broad scale and local information about climate risk to National Meteorological Services in 15 partner countries. Investing development time in open-source geospatial data infrastructure and re-usable user interface frameworks has enabled rapid development of new tools for forecasts of new predictands. Extension of prediction research to the responsible agencies has involved training of users and continuing support.
\end{abstract}

Keywords: Seasonal prediction, Climate extremes, Dynamical climate model 


\section{INTRODUCTION}

The countries of the Pacific Ocean are exposed to climate risk across a range of sectors, most notably in water resources, agriculture and disaster preparedness. Small changes in the mean state of the climate system can lead to large changes in the frequency and magnitude of extreme events (Corti et al., 1999). A recently completed global analysis has found a near 50-fold increase in the frequency of extremely hot temperatures during the northern hemisphere summer (Hansen et al., 2012). Evidence is accumulating to support the hypothesis that global warming has intensified the hydrological cycle, causing more extreme flooding and droughts (Huntington. 2006). In this changing climate, the historical frequencies of extreme events such as droughts, tropical cyclones and coastal inundation are less useful as predictive probabilities (Rahmstorf and Coumou, 2011).

Considerable uncertainties remain about the impact of climate change on extreme weather events. In the face of these uncertainties, an effective and low cost option to reduce vulnerability to climate change is to improve the accuracy, availability and use of forecasts (Lubchenco and Karl, 2012).

While many climate anomalies are essentially chaotic and not predictable, the large-scale coupling between the atmosphere and the ocean imparts a degree of predictability to variations of seasonal climate in the atmosphereocean-land surface system. The most significant manifestation of this coupling, and the physical source of much of this predictability is the El Niño Southern Oscillation (ENSO). This predictability is utilised by coupled ocean-atmosphere General Circulation Models (CGCMs) which form the basis of modern seasonal forecasting systems. Integral to the forecast capability of such models are ocean and atmospheric data assimilation systems, which provide an estimate of the state of the system as measured by satellite observations, buoys such as the TOGA-TAO in the equatorial Pacific Ocean and other instruments.

In this paper, the CGCM-based seasonal prediction system is described. Experimental outlooks for extreme sea levels, extreme ocean temperatures, seasonal tropical cyclone activity, rainfall and temperature are then described. Finally the web application used to deliver the outlooks to users in National Meteorological and Hydrological Services (NMSs) is described.

\section{DYNAMICAL MODEL PROBABILITY OUTLOOKS}

The operational Predictive Ocean Atmosphere Model for Australia 'multi-week' (POAMA-M24) system is described in detail in a Bureau of Meteorlogoy technical bulletin (National Meteorological and Oceanographic Centre, 2013). It comprises a T47 spectral atmospheric model with 17 vertical levels, coupled using the Ocean Atmosphere Sea Ice Soil coupler (OASIS) to an ocean model with 25 vertical levels, $2^{\circ}$ zonal resolution and $0.5^{\circ}$ meridional resolution at the equator increasing to $1.5^{\circ}$ near the poles. Land surface initial conditions are generated by a nudging scheme. An ensemble of 10 ocean and atmospheric states perturbed around a central analysis is generated using a coupled breeding scheme (Hudson et al., 2013).

Seasonal climate prediction is inherently probabilistic because the evolution of the climate system is highly sensitive to initial conditions. The nonlinear nature of the coupled ocean-atmosphere system means that probabilities of extreme events may not be well estimated from a single best guess deterministic forecast. To estimate the range of physically plausible outcomes, GCMs are typically run as an ensemble with perturbed initial conditions. The economic value of ensemble forecasts is greater than that of individual models or simple ensemble means (Palmer and Räisänen, 2002).

Because the numerical integration of equations of motion does not change under global warming, dynamical models are less influenced by climate change than statistical models. GCMs explicitly take into account climate processes that are important for seasonal climate prediction such as equatorial oceanic waves and atmospheric convection driven by ocean temperatures and are not constrained by what has occurred in the past. GCMs implicitly include the effects of a changing climate whatever its character or cause and can predict outcomes not seen previously. Dynamical models are of course subject errors and biases, and to the fundamental limits to predictability of the ocean-atmosphere system. Such errors and limitations are assessed over a hindcast (retrospective forecast) period in order to provide information about the likely skill of the outlooks.

\section{Sea LeVel}

Sea level rise is expected to have some of the most profound impacts on islands in the Western Pacific through coastal erosion, damage to physical assets and loss of agricultural productivity due to salt water intrusion and increasing salinity. 
a)

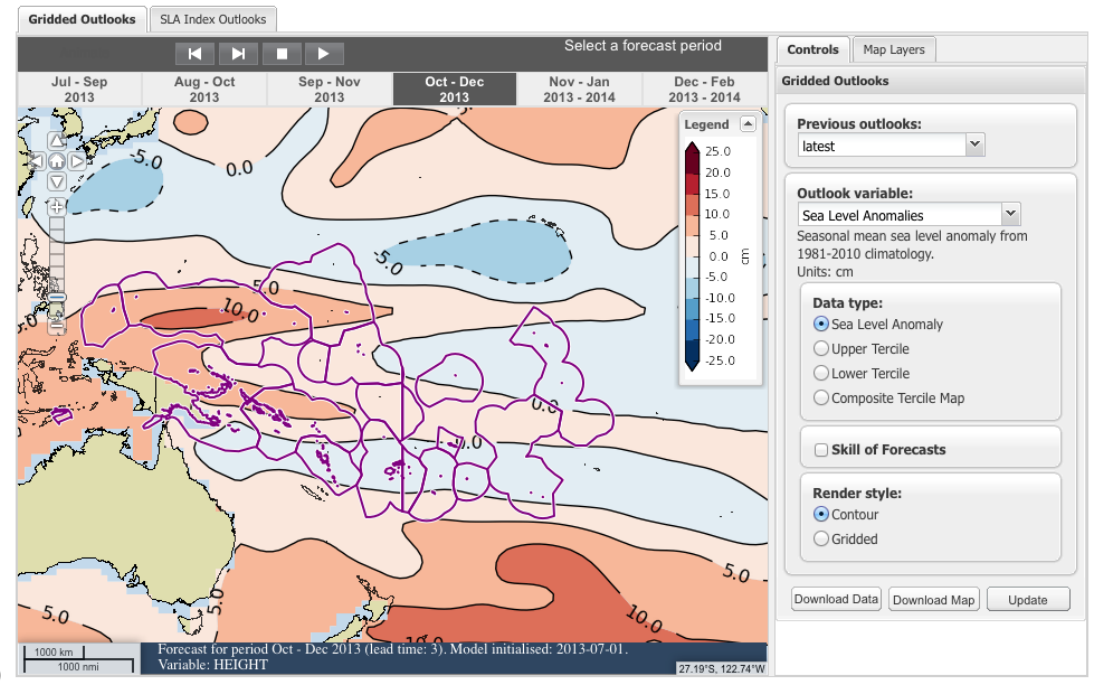

b)

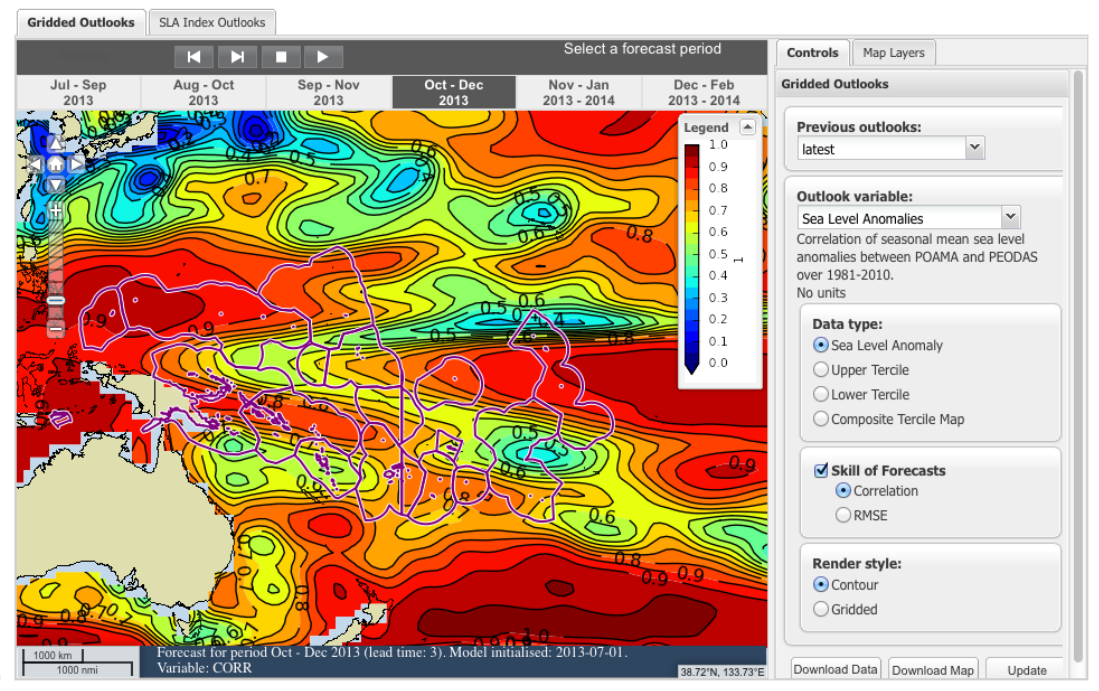

Figure 1. a) Sea level anomaly outlook for October to December 2013, published at http://poama.bom.gov.au/ experimental/pasap/sla.shtml. b) Correlation skill for October to December predictions of three monthly mean sea level anomalies,

Outlooks for three monthly mean sea level anomalies, and of the probability of seasonal sea levels falling into the lowest or highest tercile, are generated directly from the POAMA-M24 ocean model ensemble. Forecasts at a range of lead times are published, along with the associated verification scores. Figure 1 1 a shows an example forecast, while Figure $1 \mathrm{p}$ shows the correlation coefficient between the model outlook for three monthly mean sea level anomaly and a realtime analysis of sea level (Yin et al., 2011). Hindcast analysis shows that at short lead times the sea level anomaly outlooks are quite skillful as shown in Figure $1 \mathrm{p}$. The sea level outlooks are described further in (Miles et al., 2013).

\section{OCEAN TEMPerature}

Coral bleaching is largely a result of anomalously warm water temperatures. Degraded coral reefs present many potential social and economic problems for Pacific island countries, including long-term loss of tourism, potential loss or degradation of fisheries and reduction in coastal protection, as well as the environmental degradation itself. Monitoring and prediction of thermal stress events enables the support of adaptive and management activities that could improve reef resilience to extreme conditions. Forecasts of the probability of temperatures exceeding key coral sensitivity thresholds can be used to plan reef management activities. 
a)
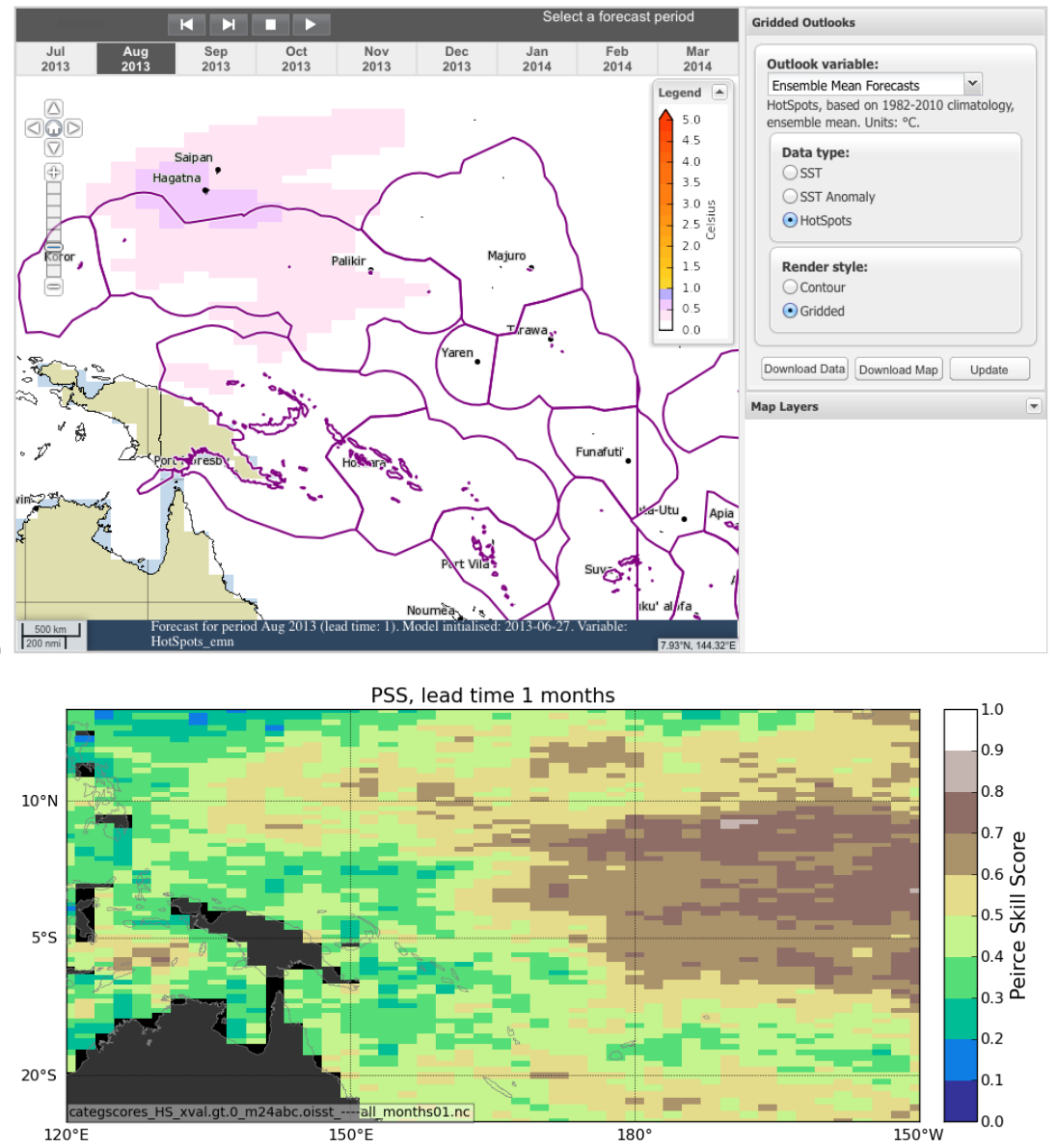

b)

Figure 2. a) Outlook for 'HotSpots' (positive temperature anomalies), displayed at http://poama.bom.gov.au/ experimental/pasap/sst.shtml b) Pierce skill score for POAMA HotSpot outlooks.

Griesser and Spillman (2012) present an assessment of the predictive skill of the POAMA-M24 system for ocean temperature. Figure 2 a shows an outlook for 'HotSpots', regions of positive temperature anomalies. Figure $2 \mathrm{p}$ shows the Pierce skill score for categorical forecasts of HotSpots. The Pierce score is a categorical skill score based on the false alarm rate and hit rate, where 0 indicates no skill and 1 indicates perfect forecasts. This information enables users of the outlooks to take historical skill into account when using the forecasts for decision-making.

\section{Tropical Cyclones}

Tropical Cyclones (TCs) are the most destructive weather systems that impact on coastal areas across the Pacific. Currently, seasonal outlooks of the likelihood of extreme, synoptic timescale events such as tropical cyclones are used for disaster preparedness. While individual tropical cyclones are not predictable beyond timescales of the order of one week, the distribution of tropical cyclone activity is influenced by large-scale climatic features such as ENSO. When such predictable shifts in TC activity are captured by GCMs, useful forecasts of TC activity for the season ahead can be made. Such long range forecasts enable disaster management resources to be allocated ahead of time, providing a complement to the shorter timescale forecasts of the formation and development of individual TCs.

Figure 3 shows the time series of the number of TCs detected in an integration of the POAMA model over October to April for the period 1982 to 2010, in the South Pacific and Australian regions. The correlation of 0.5 between ensemble mean and the observed storm count in the South Pacific demonstrates a modest ability to predict variability associated with ENSO. The ensemble spread is appropriate to the outlook uncertainty. 

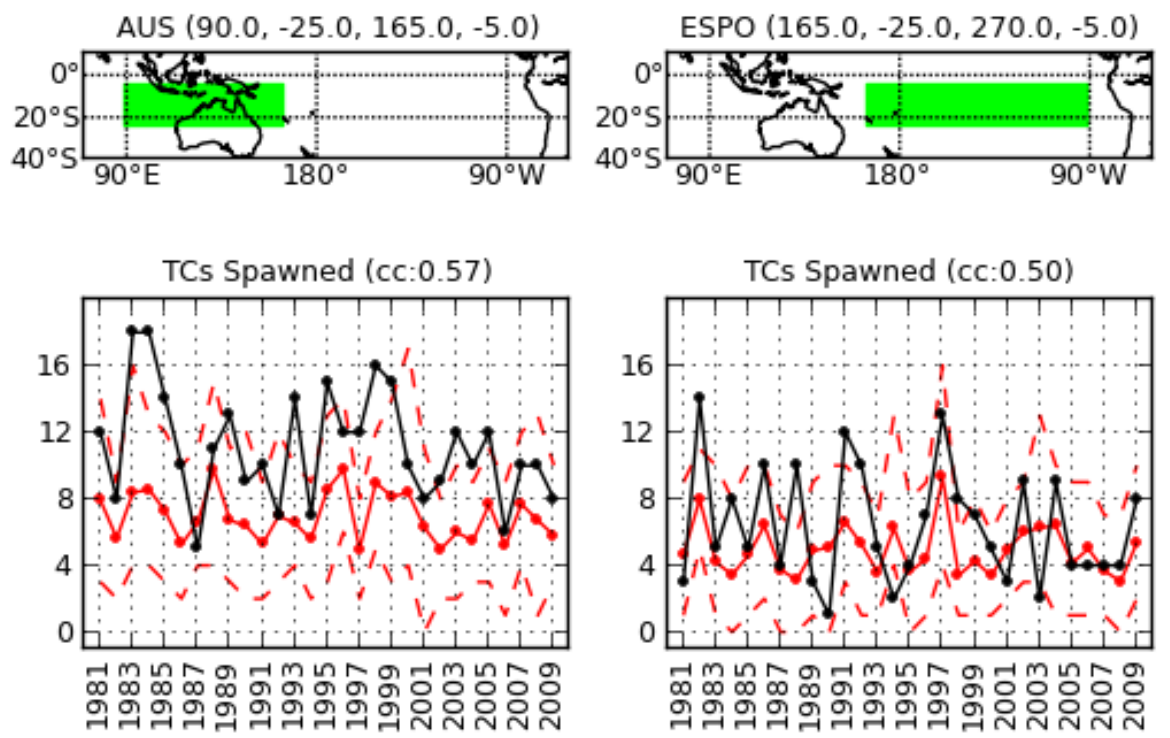

Figure 3. Time series of numbers of tropical cyclones in Australian and eastern South Pacific regions per year. Red: $\min / \max$ and ensemble mean TC counts from the POAMA-M24 seasonal prediction system. Black: observed counts from southern hemisphere tropical cyclone archive (Kuleshov et al., 2010). For the Australian region, the correlation coefficient between observed and modelled counts is 0.57 . For the eastern South Pacific it is 0.5 .
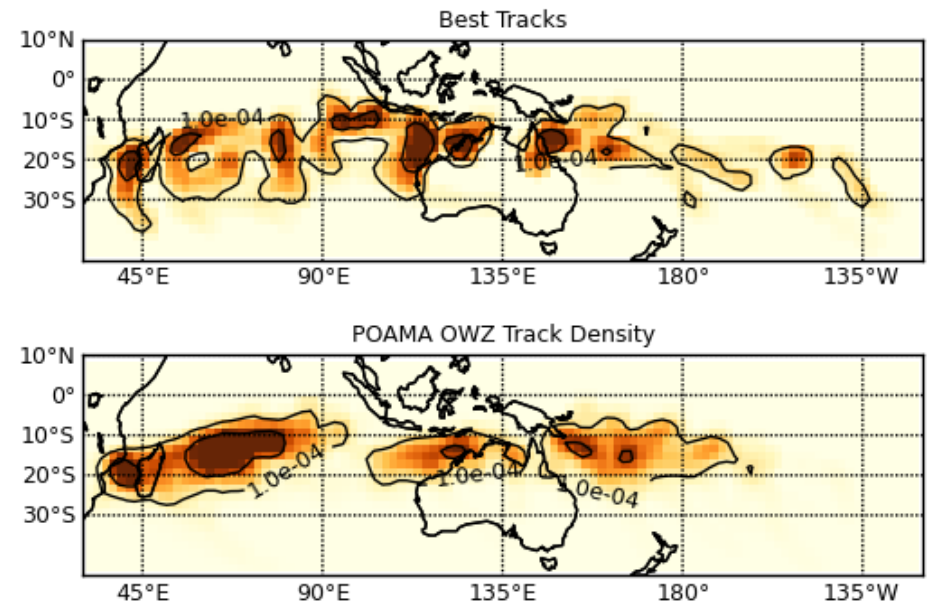

Figure 4. Top: Observed TC tracks in the Southern Hemisphere in 1985. Bottom: TC track density from ten ensemble members of the POAMA system. Units are storms/area, normalised.

Figure 4 shows observed Southern Hemisphere tracks for 1985, compared to the track density computed over an ensemble of ten model runs of the POAMA seasonal forecasting system. This track density is calculated using a radially symmetric fourth order polynomial smoothing kernel with a cutoff of approximately $500 \mathrm{~km}$. 
The use of this smoothing enables the ensemble of forecasts to be presented as a field, summarising the risk of storms in particular areas.

\section{RAINFALL AND TEMPERATURE}

Rainfall and temperature tercile probability outlooks are presented at monthly climate outlook forum meetings involving staff from NMSs across the Pacific region (Cottrill et al., 2013). The provision of an ensemble mean forecast provides a further indication of potential extreme conditions such as drought and flood. With additional work this could be extend to quantitative predictions of extreme categories of rainfall.

\section{USER INTERFACES, TOOLS AND INFRASTRUCTURE}

These predictions of climate extremes are delivered via web-based applications which display the outlooks as overlays on navigable maps. The map allows outlooks for a variety of predictands along with information about outlook skill to be selected and displayed. Time series information is displayed for sites or regions via their selection on the map. Contextual information is displayed and forecast data and figures can be downloaded.

Two factors driving innovation in the software used to distribute climate forecasts are the increasing amount of forecast data and expectations from users for richer applications. The amount of gridded and time series forecast data made available by dynamical models is large and increasing. At the same time geospatial software frameworks are developing rapidly. Users demand a richer experience and developers expect consistent application programming interfaces (APIs) for accessing and using data.

The software tools described above provide this rich experience. In the design of our system architecture we incorporated two key design principles: the use of open standards and interoperable sub-systems. Four distinct layers are defined as components of the overall process of turning the outputs of GCMs into seasonal outlooks suitable for use by decision-makers. This is a generalisation of the software architecture for seasonal forecast production and delivery described in Charles et al. (2011).

The model layer comprises the POAMA seasonal prediction system, simulating the evolution of the coupled ocean-atmosphere system and the systems for the ingestion of observational data and assimilation of these observations into the model, the construction of an ensemble of initial states and the output of variables of interest.

In the forecast generation layer, forecast products are generated from dynamical model output. This process involves statistical corrections for model biases and may involve the use of outputs from different models. Analysis of model performance over a historical period guides the decisions made at this level. The resulting derived forecast products are stored in self-describing files with additional metadata. Careful metadata curation in this layer enables downstream users of the data to understand its provenance and is considered a best practice for public data.

At the data service layer forecast data is exposed using interfaces such as OPEnDAP (Cornillon et al., 2003). The format of the output is not dependent on the particular dynamical model, or even that the model is dynamical: the forecast is simply a time series of gridded data with descriptive metadata. Clearly defined APIs for data access reduce the burden on application and product developers.

The outlook product layer provides the means for the majority of users to access forecasts, typically in the form of maps and graphs presented as images, data tables and expert commentary. The application presenting the extremes outlooks uses the BOM.Map JavaScript mapping library and Python based web services generating artifacts such as images and data tables. The objective is to present a seamless interface that hides the technical complexity from the end user, providing direct access to future climate information.

The climate extremes outlook tools described above were presented at a capacity building science symposium in Honiaria in February 2013, attended by representatives from 14 Pacific Island and East Timor NMSs, and subsequently at a training workshop for NMS staff. NMS staff are involved in ongoing work to verify the outlooks against observations.

\section{CONCLUSIONS}

The potential of dynamical seasonal forecasting for providing foresight of extreme climatic events has been explored, and developed into a suite of experimental forecast products targetted at Pacific NMSs. Much work remains to be done in connecting decision-makers with information, and in the research and development of outlooks for other extremes. Seasonal predictions are published experimentally at the following URLs: 
- Sea level predictions: http://poama.bom.gov.au/experimental/pasap/sla.shtml

- Extreme ocean temperature predictions: http://poama.bom.gov.au/experimental/pasap/sst.shtml

- Rainfall and temperature (WMO Global Producing Centre for Long Range Forecasts, Melbourne): http://poama.bom.gov.au/experimental/pasap/

\section{ACKNOWLEDGEMENT}

The research discussed in this paper was conducted with the support of the Pacific Australia Climate Change Science and Adaptation Planning Program (PACCSAP), a program supported by AusAID, in collaboration with the Department of Climate Change and Energy Efficiency, and delivered by the Bureau of Meteorology and the Commonwealth Scientific and Industrial Research Organisation (CSIRO).

\section{REFERENCES}

Charles, A., D. McClymont, R. de Wit, and D. Jones (2011). A software architecture for seasonal climate forecasts in the tropical Pacific. In In Chan, F., Marinova, D. and Anderssen, R.S. (eds) MODSIM2011, 19th International Congress on Modelling and Simulation. Modelling and Simulation Society of Australia and New Zealand, December 2011, pp. 1188-1194.

Cornillon, P., J. Gallagher, and T. Sgouros (2003). OPeNDAP: accessing data in a distributed, heterogeneous environment. Data Science Journal 2, 164-174.

Corti, S., F. Molteni, and T. N. Palmer (1999, April). Signature of recent climate change in frequencies of natural atmospheric circulation regimes. Nature 398(6730), 799-802.

Cottrill, A., H. H. Hendon, E.-P. Lim, S. Langford, K. Shelton, A. Charles, D. McClymont, D. Jones, and Y. Kuleshov (2013, February). Seasonal forecasting in the Pacific using the coupled model POAMA-2. Weather and Forecasting, 130225133541001.

Griesser, A. and C. Spillman (2012). SST forecast skill of the new intra-seasonal configuration of POAMA-2. CAWCR Research Letters Issue 8 (8), 10-16.

Hansen, J., M. Sato, and R. Ruedy (2012, August). Perception of climate change. Proceedings of the National Academy of Sciences.

Hudson, D., A. G. Marshall, Y. Yin, O. Alves, and H. H. Hendon (2013, July). Improving intraseasonal prediction with a new ensemble generation strategy. Monthly Weather Review, 130731140004007.

Huntington, T. G. (2006, March). Evidence for intensification of the global water cycle: Review and synthesis. Journal of Hydrology 319(1-4), 83-95.

Kuleshov, Y., R. Fawcett, L. Qi, B. Trewin, D. Jones, J. McBride, and H. Ramsay (2010). Trends in tropical cyclones in the south Indian Ocean and the south Pacific Ocean. Journal of Geophysical Research: Atmospheres 115(D1).

Lubchenco, J. and T. R. Karl (2012). Predicting and managing extreme weather events. Physics Today 65(3), 31.

Miles, E., C. M. Spillman, J. A. Church, and P. C. Mcintosh (2013). Seasonal prediction of global sea-level anomalies using an ocean-atmosphere dynamical model. In preparation.

National Meteorological and Oceanographic Centre (2013, March). Operational upgrade to Predictive Ocean Atmosphere Model for Australia (POAMA-M24). NMOC Operations Bulletin 96, Bureau of Meteorology.

Palmer, T. N. and J. Räisänen (2002, January). Quantifying the risk of extreme seasonal precipitation events in a changing climate. Nature 415(6871), 512-514.

Rahmstorf, S. and D. Coumou (2011, November). Increase of extreme events in a warming world. Proceedings of the National Academy of Sciences 108(44), 17905-17909.

Yin, Y., O. Alves, and P. R. Oke (2011, March). An ensemble ocean data assimilation system for seasonal prediction. Monthly Weather Review 139(3), 786-808. 